\begin{tabular}{|c|c|}
\hline Title & $\begin{array}{l}\text { Fundamental study on filter effect of confronting divergent magnetic fields applied to low-pressure inductively coupled } \\
\text { plasmas }\end{array}$ \\
\hline Author(s) & Sugaw ara, Hirotake; Ogino, So \\
\hline Citation & $\begin{array}{l}\text { Japanese Journal of A pplied Physics, 55(7S2), 07L D05 } \\
\text { https://doi.org/10.7567/JJA P.55.07LD 05 }\end{array}$ \\
\hline Issue Date & $2016-06-13$ \\
\hline Doc URL & http:/hdl. handle.net/2115/66168 \\
\hline Rights & (c) 2016 The Japan Society of A pplied Physics \\
\hline Type & article (author version) \\
\hline File Information & Sugawara 2016-JJA P-HU SCA P.pdf \\
\hline
\end{tabular}

Instructions for use 


\title{
Fundamental study on filter effect of confronting divergent magnetic fields applied to low-pressure inductively coupled plasmas *
}

\author{
Hirotake SugAWARA $^{\dagger}$ and So OGINO \\ Graduate School of Information Science and Technology, Hokkaido University, Sapporo 060-0814, \\ Japan
}

The electron motion under confronting divergent magnetic fields (CDMFs) applied to inductively coupled plasmas was simulated using a Monte Carlo method. The CDMFs induced by two coaxial dc coils confined electrons in one side of the separatrix of the CDMFs. However, electrons diffused across the separatrix mainly in two ways. One was the displacement of their gyrocenters due to scattering near the outer part of the separatrix. This process tended to occur for high-energy electrons with correspondingly large gyroradii. The other was passage through the weak magnetic field around the center. This process was allowed for low-energy electrons. The position-dependent selectivity about the electron energy was pointed out as a seed property for applications of the separatrix as a magnetic filter or shutter. The mechanism by which the functions of the separatrix emerge was explained from a viewpoint of electron motion under the CDMFs.

\section{Introduction}

A magnetic field separates chosen charged particles out of a plasma flow. This function has often been referred to as a magnetic filter effect. Typical magnetic filters are, e.g., curved coils to guide chosen species ${ }^{1-5}$ and magnetic barriers to section a downstream region from an upstream plasma source. ${ }^{6-11}$ The filter effect of confronting divergent magnetic fields (CDMFs), presented in this paper, is unique such that it appears at a particular geometrical structure called the separatrix.

The CDMFs are induced by two coaxial coils with loop dc currents flowing in opposite directions (see Fig. 1). The CDMFs have a cusp structure at their separatrix and a magnetic null point called the $\mathrm{X}$ point at the intersection between the axis and the separatrix. The magnetic field strength increases outward with the distance from the $\mathrm{X}$ point. The X-point plasma $^{12-15}$ proposed as a hydrogen negative ion source is an inductively coupled plasma driven under the CDMFs. It utilizes the filter effect of the separatrix to limit the plasma enhancement region. At the separatrix, a steep change in the profile of optical emission from the plasma was observed as a filter effect. ${ }^{14}$

As potential applications of the filter effect of the separatrix, we may consider, e.g., down-

\footnotetext{
*Published source: Japanese Journal of Applied Physics 55, 07LD05-1 - 07LD05-6 (2016)

${ }^{\dagger}$ E-mail: sugawara@ist.hokudai.ac.jp
} 


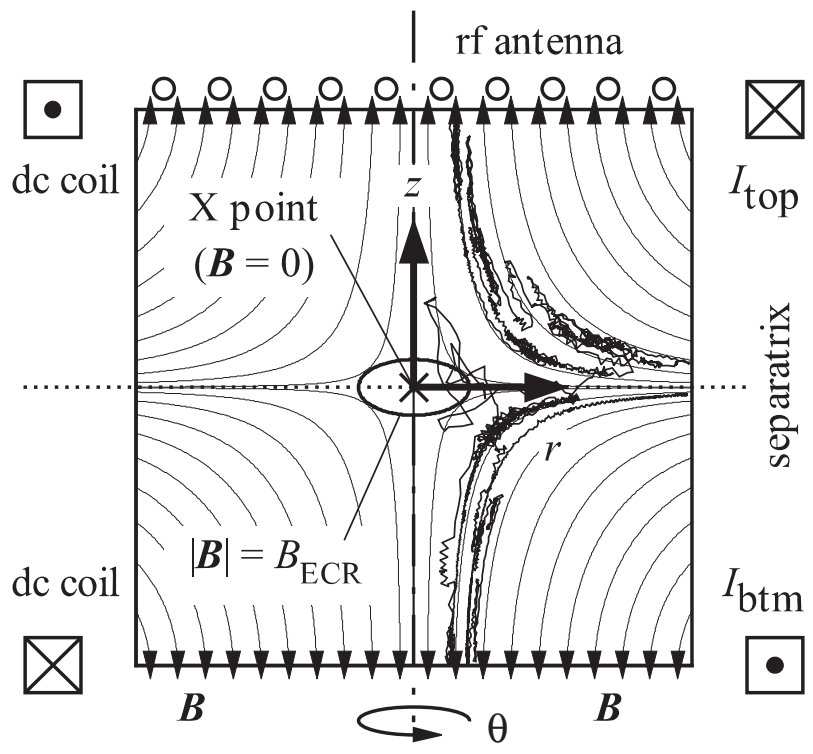

Fig. 1. Schematic of a model reactor and an example of electron locus under CDMFs. The CDMFs induced by the top and bottom coils have a magnetic null point ( $\mathrm{X}$ point) at the center and a separatrix at $z=0$. The electron locus was calculated assuming $\mathrm{H}_{2}$ at $1.0 \mathrm{~Pa}, I_{\text {top }}=+50.0 \mathrm{~A}$, $I_{\mathrm{btm}}=-50.0 \mathrm{~A}$, and an antenna current $-10 \cos 2 \pi f_{\mathrm{rf}} t \mathrm{~A}$ at $f_{\mathrm{rf}}=13.56 \mathrm{MHz}$.

sizing of remote plasmas and promotion of volume reactions for negative ion etchant production by selectively supplying low-energy electrons. However, features of electron transport across the separatrix have rarely been reported in detail. This is in contrast to the fact that the guide of charged particles along the separatrix is well studied in applications of diverters to capture impurity particles in tokamak reactors ${ }^{16}$ and uniform irradiation of etchant ions to semiconductor wafers in processing plasmas under a quadrupole magnetic field. ${ }^{17-21}$

In this paper, we present results of Monte Carlo simulations on the behavior of electrons driven by an rf electric field under the CDMFs. We point out some characteristic effects of the separatrix on the electron transport such as electron confinement and energy-selective electron passage, which are expected as seeds of the applications mentioned above. We analyze the mechanism by which the filter effect of the separatrix emerges.

\section{Simulation model and conditions}

The simulation results presented in this paper were obtained by Monte Carlo simulations. Desirable conditions were chosen to demonstrate specific features of the electron behavior under the CDMFs. Common assumptions in the model are explained here, and particular parameters are shown when each result is presented. 


\subsection{Reactor geometry}

The reactor assumed in the present work is shown in Fig. 1. The chamber was a cylinder with a $20.0 \mathrm{~cm}$ inner radius and a $40.0 \mathrm{~cm}$ height. The origin of position $(x, y, z)$ or $(r, \theta, z)$ was defined at the center of the chamber. Here, $x=r \cos \theta$ and $y=r \sin \theta$. The CDMFs, $\boldsymbol{B}$, were applied by two 36-turn dc coaxial coils with a cross section of $4.0 \times 4.0 \mathrm{~cm}^{2}$. The inner and outer radii of the coils were 24.0 and $28.0 \mathrm{~cm}$, respectively. The coils were basically aligned at $z= \pm 20.0 \mathrm{~cm}$, i.e., the levels of the chamber ceiling and bottom, and were shifted upward in parallel in a demonstration of the electron confinement effect.

\subsection{Electric and magnetic fields}

The dc currents of the top and bottom coils used to apply $\boldsymbol{B}$ were set as $I_{\mathrm{top}}=+I_{\mathrm{dc}}$ and $I_{\mathrm{btm}}=-I_{\mathrm{dc}}$ to form a plane separatrix. $I_{\mathrm{dc}}$ was varied in the range of $33.3-100.0 \mathrm{~A}$. $\boldsymbol{B}$ was assumed to have only the radial and axial components, $B_{r}$ and $B_{z}$, respectively.

The rf electric field $\boldsymbol{E}$ was induced by a planar spiral antenna on top of the chamber with current $I_{\text {antenna }}(t)=-I_{\mathrm{rf}} \cos 2 \pi f_{\mathrm{rf}} t$. The frequency and amplitude were $f_{\mathrm{rf}}=13.56 \mathrm{MHz}$ and $I_{\mathrm{rf}}=10.0-30.0 \mathrm{~A}$, respectively. The antenna current was, for simplicity, approximated by concentric circular currents flowing at $r=2.0,6.0,10.0,14.0$, and $18.0 \mathrm{~cm}$ and $z=21.5 \mathrm{~cm}$. $\boldsymbol{E}$ was then treated to have only the azimuthal component $E_{\theta}$ represented as $E_{\theta}(r, z, t)=$ $E_{\max }(r, z) \sin 2 \pi f_{\text {rf }} t$.

The position-dependent values of $B_{r}, B_{z}$, and $E_{\max }$ were calculated on the basis of the Biot-Savart law and Faraday's law of induction. The $r-z$ distributions of $E_{\max }$ and $|\boldsymbol{B}|$ are shown in Fig. 2. Their peak values per unit current are $0.898 \mathrm{~V} / \mathrm{cm}$ at $I_{\mathrm{rf}}=1.0 \mathrm{~A}$ and $0.166 \mathrm{mT}$ at $I_{\mathrm{dc}}=1.0 \mathrm{~A}$, respectively. The temporal variation of $\boldsymbol{B}$ induced by the $\operatorname{rf} \boldsymbol{E}$ field was neglected because it was smaller than the dc component of $\boldsymbol{B}$ by about four to five orders of magnitude. Some previous simulations by the authors' group considered the space charge field, ${ }^{22,23}$ but it was not considered here to highlight the direct effect of the CDMFs on the electron transport.

\subsection{Trace of electrons}

The initial electrons were released from the chamber ceiling $z=20.0 \mathrm{~cm}$ with initial velocities chosen at random from a Maxwellian distribution with a mean energy of $1.0 \mathrm{eV}$. The range of $r$ for the release of the initial electrons was chosen for convenience of saving simulation time depending on the purpose of simulation. For example, most of the electrons released from a large $r$, i.e., from an outer area of the chamber ceiling, had never entered the lower region during a reasonable simulation time. Thus, a trace of such electrons was omitted in simulations to sample those crossing the separatrix.

The electron trajectory was calculated using the Runge-Kutta method for the following 


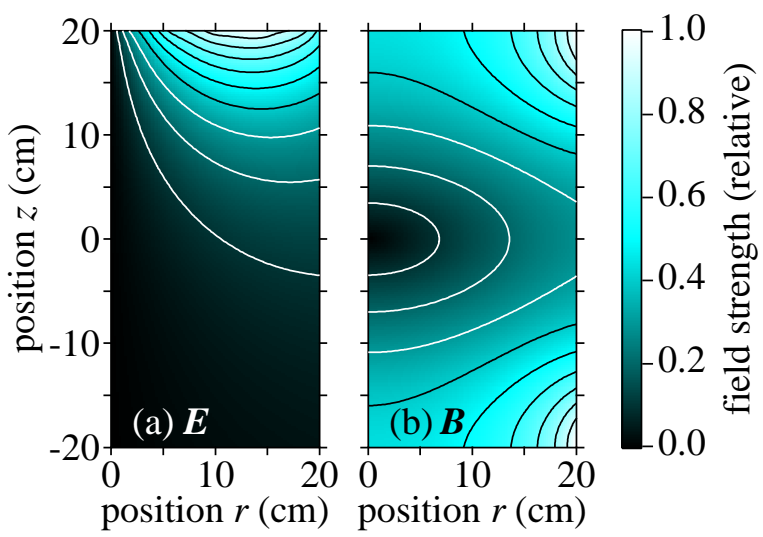

Fig. 2. (Color online) Distributions of (a) amplitude of the $\boldsymbol{E}$ field and (b) strength of the $\boldsymbol{B}$ field. The contours represent $0.10-0.90$ of the peak value with steps of 0.10 .

electron motion equations:

$$
\frac{\mathrm{d} \boldsymbol{v}}{\mathrm{d} t}=-\frac{e}{m}(\boldsymbol{E}+\boldsymbol{v} \times \boldsymbol{B}), \quad \frac{\mathrm{d} \boldsymbol{r}}{\mathrm{d} t}=\boldsymbol{v},
$$

where $\boldsymbol{r}$ and $\boldsymbol{v}$ are the position and velocity of an electron, and $e$ and $m$ are the electronic charge and mass, respectively. The simulation time step $\Delta t$ was $3.7 \mathrm{ps}$, which is $1 / 20000$ of the rf period $T_{\mathrm{rf}}=1 / f_{\mathrm{rf}}=73.7 \mathrm{~ns}$.

The gas was assumed to be Ar at $0.67 \mathrm{~Pa}$ or $\mathrm{H}_{2}$ at $1.0 \mathrm{~Pa}$ at $300 \mathrm{~K}$. The occurrence of the collisional events was judged by a time-saving scheme ${ }^{24}$ using random numbers on the basis of the collision probability. The probability was derived from the electron collision cross sections taken from Ref. 25 for $\mathrm{Ar}$ and Ref. 26 for $\mathrm{H}_{2}$. Each of the cross section sets for $\mathrm{Ar}$ and $\mathrm{H}_{2}$ includes processes of elastic collision, excitation, and ionization.

The electron reflectivity $r_{\text {ref }}$ at the chamber wall and the remaining energy ratio $r_{\text {remain }}$ at a reflection were set to be as high as $r_{\text {ref }}=0.99-1.00$ and $r_{\text {remain }}=0.50-1.00$ so as not to lose sample electrons during the trace. The trace time was $100 T_{\mathrm{rf}}-500 T_{\mathrm{rf}}$. This period corresponds to an early phase of the electron diffusion in the chamber.

\section{Results and discussion}

\subsection{Fundamental electron motion}

A typical electron locus under the CDMFs is shown in Fig. 1. The electron gyrated around its guiding magnetic flux lines and reciprocated along them between the chamber ceiling and the side wall. Electron reflection occurred during the reciprocation at the boundary or was induced by the magnetic mirror effect. A libration of the gyrocenter by the $\boldsymbol{E} \times \boldsymbol{B}$ drift $^{27}$ was also observed. The electron deviated from the guiding magnetic flux lines by collisional scattering, meandering in the weak $\boldsymbol{B}$ field region around the $\mathrm{X}$ point, or rf-resonant acceleration $^{23}$ near the region of $|\boldsymbol{B}|=B_{\mathrm{ECR}}$. 
Here, $B_{\mathrm{ECR}}=2 \pi f_{\mathrm{rf}}(m / e)$ is the rf-resonant magnetic field strength, at which the electron cyclotron resonance occurs. $B_{\mathrm{ECR}}=0.48 \mathrm{mT}$ at $f_{\mathrm{rf}}=13.56 \mathrm{MHz}$. The electron gyration occurs in a strong $\boldsymbol{B}$ field region, roughly $|\boldsymbol{B}|>B_{\mathrm{ECR}}$, far from the X point. On the other hand, in a weak $\boldsymbol{B}$ field region of $|\boldsymbol{B}|<B_{\mathrm{ECR}}$ around the X point, the electron gyration is incomplete and the electron trajectory becomes chaotic. ${ }^{28}$ In the present configuration, $|\boldsymbol{B}|$ on the separatrix is approximately proportional to $r$ around the $\mathrm{X}$ point. The $r$ position of $|\boldsymbol{B}|=B_{\mathrm{ECR}}$ on the separatrix, $r_{\mathrm{ECR}}$, is about $2.0,4.0$, and $6.0 \mathrm{~cm}$ at $I_{\mathrm{dc}}=100.0,50.0$, and $33.3 \mathrm{~A}$, respectively. $r_{\mathrm{ECR}}$ is to be regarded as the aperture radius when electron transport across the separatrix is considered in Sect. 3.5.

The electron was guided sideways by the magnetic flux lines. This prevented the electrons from straightforward diffusion to the lower region and appeared as a confinement effect, while electrons under $\boldsymbol{B}=0$ distributed more homogeneously in the chamber. ${ }^{29}$ However, two ways of downward diffusion were observed. ${ }^{29,30}$ One was passage through the weak $\boldsymbol{B}$ field region. The gyroradii were large there and the electron capture and guide by the magnetic flux lines were not effective. This process was unselective about electron energy, and thus, was allowed for low-energy electrons. The other was scattering near the outer part of the separatrix. Electron transfer from guiding magnetic flux lines running in the upper region to those in the lower region occurred with the displacement of the gyrocenters of the scattered electrons. This process seemed likely to occur for high-energy electrons with correspondingly large gyroradii. These tendencies are verified in Sect. 3.5 by statistics of electrons passing across the separatrix.

\subsection{Electron confinement by separatrix}

Figure 3 shows aspects of electron confinement in $\mathrm{Ar}$ at $0.67 \mathrm{~Pa}$ under strong and weak CDMFs at $I_{\mathrm{dc}}=100.0$ and $33.3 \mathrm{~A}$, respectively. 50000 electrons were released from the chamber ceiling uniformly. The electron concentration $n_{\mathrm{e}}$ was time-averaged during $t=400 T_{\mathrm{rf}^{-}}$ $500 T_{\text {rf }}$.

Under the strong CDMFs, in Figs. 3(a)-3(f), the electron confinement by the separatrix worked well. Most of the electrons were confined in the upper region up to $500 T_{\text {rf }}$. Only a small fraction of them diffused downward and distributed around the central axis in the lower region.

In contrast, under the weak CDMFs, in Figs. 3(g)-3(l), the electron confinement broke as the separatrix approached the chamber ceiling. Electrons diffused to and distributed widely in the lower region. In Fig. 3(1), the peak of $n_{\mathrm{e}}$ is in the lower region, i.e. electrons in the lower region did not return to the upper region. This indicates a reverse-blocking effect of the separatrix. After electrons transferred to the lower region across the separatrix, they hardly obtained energy to cross the separatrix upward for the distance from the rf antenna. The electron confinement by the separatrix worked also in the opposite direction and the electron 

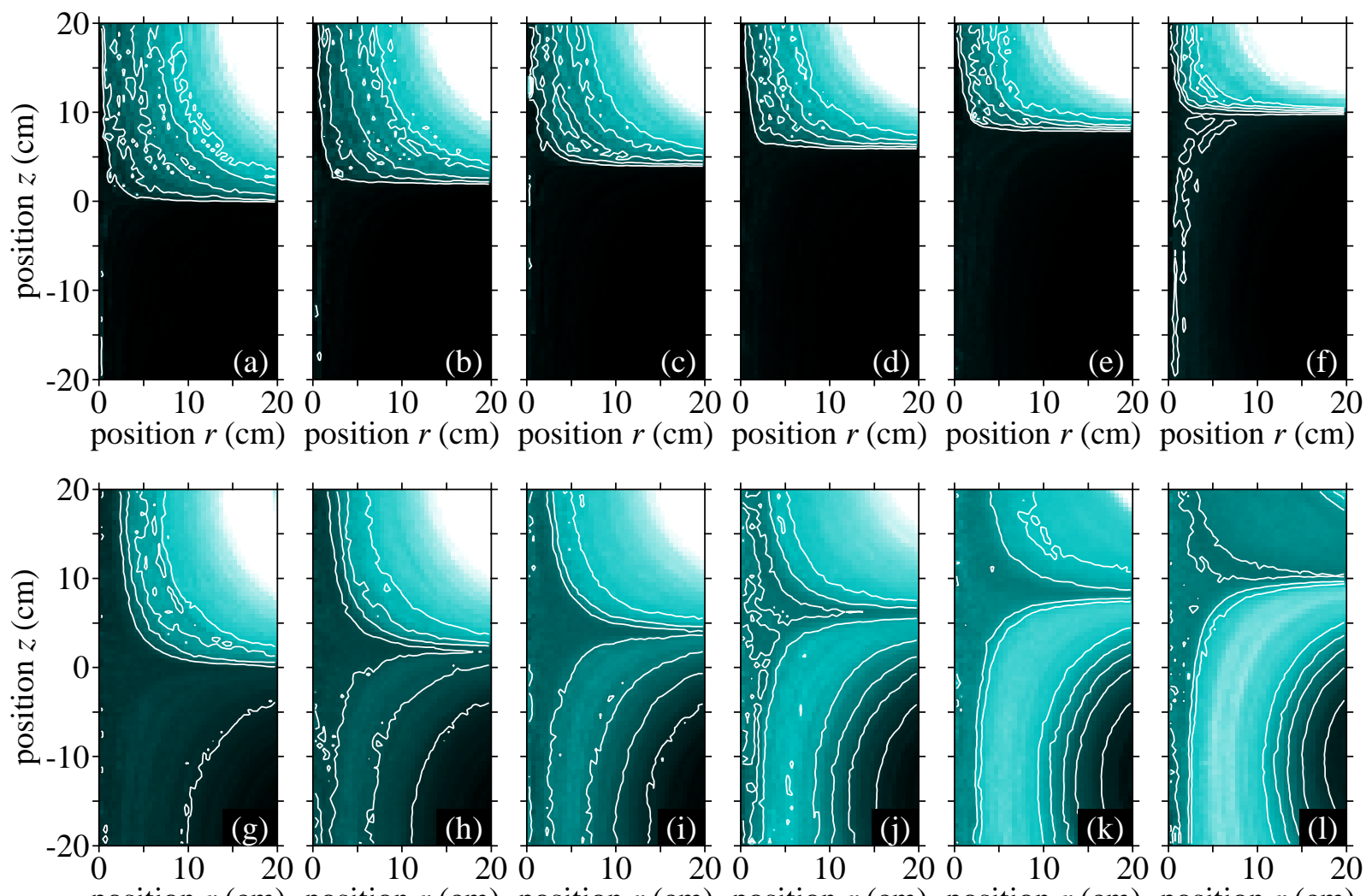

position $r(\mathrm{~cm})$ position $r(\mathrm{~cm})$ position $r(\mathrm{~cm})$ position $r(\mathrm{~cm})$ position $r(\mathrm{~cm})$ position $r(\mathrm{~cm})$

Fig. 3. (Color online) Electron concentration $n_{\mathrm{e}}(r, z)$ (number per volume, arb. unit) under CDMFs in $\mathrm{Ar}$ at $0.67 \mathrm{~Pa}$. (a)-(f) $I_{\mathrm{dc}}=100.0 \mathrm{~A}$ and $(\mathrm{g})-(\mathrm{l}) I_{\mathrm{dc}}=33.3 \mathrm{~A}$. The height of the separatrix, $z_{\mathrm{sep}}$, was set at (a) and (g) $0.0 \mathrm{~cm},(\mathrm{~b})$ and (h) $2.0 \mathrm{~cm},(\mathrm{c})$ and $(\mathrm{i}) 4.0 \mathrm{~cm},(\mathrm{~d})$ and (j) $6.0 \mathrm{~cm},(\mathrm{e})$ and (k) $8.0 \mathrm{~cm}$, and (f) and (l) $10.0 \mathrm{~cm} . n_{\mathrm{e}}(r, z)$ increases from black to white. The contours were drawn for low values of $n_{\mathrm{e}}$ up to half of the peak value to show low-concentration parts. $I_{\mathrm{rf}}=10.0 \mathrm{~A}$, $r_{\text {ref }}=1.00$, and $r_{\text {remain }}=1.00$.

passage across the separatrix was observed to depend on electron energy.

\subsection{Distributions of electrons, mean energy, and energy density}

Figure 4 shows the $r-z$ distributions of electrons $n_{\mathrm{e}}$, their mean energy $\varepsilon$, and the energy density $n_{\mathrm{e}} \varepsilon$ for 100000 electrons released uniformly from the chamber ceiling in $\mathrm{H}_{2}$ at $1.0 \mathrm{~Pa}^{29}$ They were time-averaged during a period of $100 T_{\mathrm{rf}}$ from $t=400 T_{\mathrm{rf}}$ to $500 T_{\mathrm{rf}}$.

The appearances of $n_{\mathrm{e}}(r, z), \varepsilon(r, z)$, and $n_{\mathrm{e}}(r, z) \varepsilon(r, z)$ were different from each other. $n_{\mathrm{e}}$ was high around the edge of the chamber ceiling. This was because of electron capture by a strong $\boldsymbol{B}$ field. In addition, the magnetic flux lines running between the chamber ceiling and the side wall were short there. The short electron reciprocation along the magnetic flux lines resulted in a high concentration. On the other hand, $\varepsilon$ was high in the weak $\boldsymbol{B}$ field around the $\mathrm{X}$ point. This region was far from the rf antenna, but rf-resonant electron energy gain ${ }^{23}$ occurred around the position of $|\boldsymbol{B}|=B_{\mathrm{ECR}}$ as depicted in Sect. 3.4. The peak of $\varepsilon$ was close 


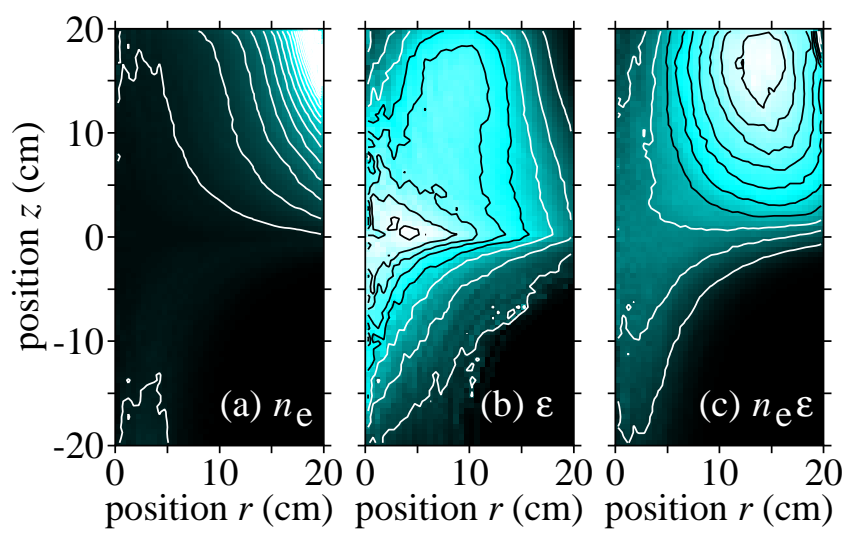

Fig. 4. (Color online) Distributions of (a) electrons $n_{\mathrm{e}}(r, z)$ (number per volume, arb. unit), (b) mean electron energy $\varepsilon(r, z)$ (contour steps: $0.2 \mathrm{eV}$; peak value: $1.65 \mathrm{eV}$ ), and (c) energy density $n_{\mathrm{e}}(r, z) \varepsilon(r, z)$ (arb. unit) in $\mathrm{H}_{2}$ at $1.0 \mathrm{~Pa}$. The values increase from black to white. $I_{\mathrm{dc}}=50.0 \mathrm{~A}$ and $I_{\mathrm{rf}}=10.0 \mathrm{~A}$. $r_{\text {ref }}=1.00$ and $r_{\text {remain }}=1.00$.

to $r=r_{\mathrm{ECR}}=4.0 \mathrm{~cm}$ on the separatrix.

It was $n_{\mathrm{e}}(r, z) \varepsilon(r, z)$ that showed a similar appearance to an optical emission profile observed in an experiment. ${ }^{14} n_{\mathrm{e}} \varepsilon$ was high below the $\mathrm{rf}$ antenna, around which $E_{\text {max }}$ takes its peak value. $n_{\mathrm{e}} \varepsilon$ is considered as a quantity applicable for a rough estimation of reaction rates of inelastic processes such as excitation and resulting optical emission because they are dependent on both $n_{\mathrm{e}}$ and $\varepsilon$.

Figure 5 shows the Abel-transformed distribution $I(x, z)$ of electron energy density $n_{\mathrm{e}}(r, z) \varepsilon(r, z)$. To fit the manner of the experimental observation, ${ }^{14} I(x, z)$ was obtained as the projection of $n_{\mathrm{e}} \varepsilon$ to the $x-z$ plane assuming the axial symmetry of $n_{\mathrm{e}} \varepsilon$ as

$$
I(x, z)=\int_{-\sqrt{R^{2}-x^{2}}}^{\sqrt{R^{2}-x^{2}}} n_{\mathrm{e}}\left(\sqrt{x^{2}+y^{2}}, z\right) \varepsilon\left(\sqrt{x^{2}+y^{2}}, z\right) \mathrm{d} y,
$$

where $R$ is the chamber radius. The appearance of $I(x, z)$, especially in Fig. 5(a), is similar to the experimental result in Ref. 14 in the following points: the peak position midway of the chamber ceiling radius, a steep decay at the separatrix, and a downward extension through the weak $\boldsymbol{B}$ field region around the X point. Primary characteristics of the appearance of the X-point plasma were reproduced at $n_{\mathrm{e}}(r, z) \varepsilon(r, z)$ even with the present simplified simulation model.

\subsection{Electron energy gain}

Figure 6 shows the distribution of the net energy gain $G(r, z)$ of electrons in $\mathrm{H}_{2}$ at $1.0 \mathrm{~Pa}$. $G(r, z)$, in units of eV per rf period per electron, includes the electron energy increase by acceleration and the energy loss by deceleration and inelastic collisions. The energy transport between sampling sections accompanying electron motion was excluded. The simulation 


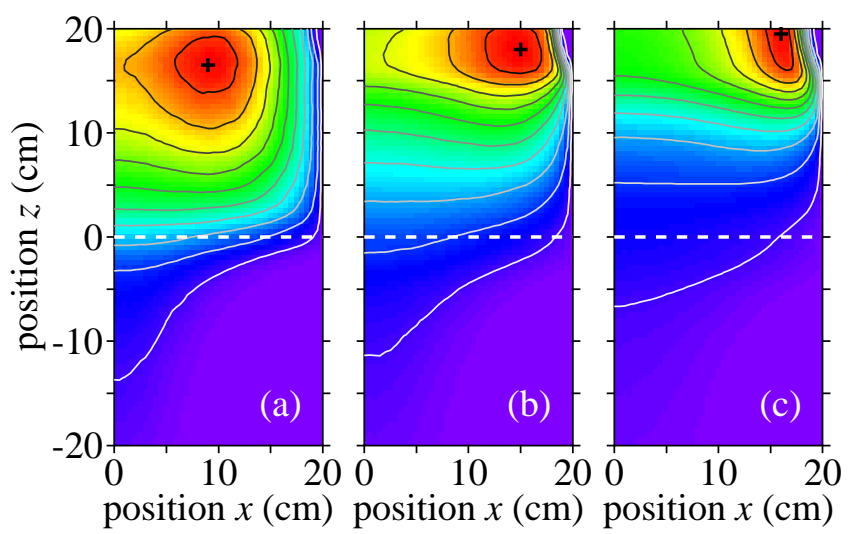

Fig. 5. (Color online) Abel-transformed distribution $I(x, z)$ of energy density $n_{\mathrm{e}} \varepsilon$ normalized by its peak value. $I_{\mathrm{rf}}=(\mathrm{a}) 10.0 \mathrm{~A}$, (b) $20.0 \mathrm{~A}$, and (c) $30.0 \mathrm{~A} . \mathrm{H}_{2}$ at $1.0 \mathrm{~Pa}, I_{\mathrm{dc}}=50.0 \mathrm{~A}$, and $t=500 T_{\mathrm{rf}}$. The + symbols indicate the peak positions. The contours represent $0.05-0.95$ of the peak value with steps of 0.10 . The values decrease with distance from the + symbol. The broken lines represent the separatrix.

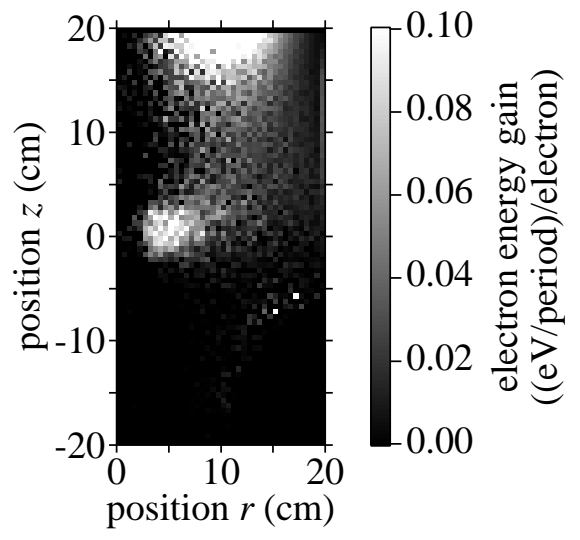

Fig. 6. Distribution of time-averaged net electron energy gain $G(r, z)$ in units of eV per rf period per electron. Only the sections of $G>0$ are depicted. $\mathrm{H}_{2}$ at $1.0 \mathrm{~Pa}, I_{\mathrm{dc}}=50.0 \mathrm{~A}$, and $I_{\mathrm{rf}}=10.0 \mathrm{~A}$. The peaks locate under the rf antenna on top of the chamber and around $(r, z)=\left(r_{\mathrm{ECR}}, 0\right)$, where $|\boldsymbol{B}|=B_{\mathrm{ECR}}$.

condition is the same as that for Figs. 1 and 4 .

One of the peaks of $G(r, z)$ appeared below the $\mathrm{rf}$ antenna, where $E_{\max }$ was high. This explains the peak position of $n_{\mathrm{e}}(r, z) \varepsilon(r, z)$. The other peak was around $(r, z)=\left(r_{\mathrm{ECR}}, 0\right)$, where $|\boldsymbol{B}|=B_{\mathrm{ECR}}$. This peak was formed by the electron cyclotron resonance. ${ }^{23}$ A similar effect was reported as partial Larmor rotation ${ }^{31}$ or partial resonance ${ }^{32}$ in nonuniform magnetic fields. However, the rf-resonant energy gain is identified neither at $n_{\mathrm{e}}(r, z) \varepsilon(r, z)$ nor in the optical emission profile ${ }^{14}$ probably for the low $n_{\mathrm{e}}$ there. If a high $\varepsilon$ is measured there experimentally, that would indirectly support the rf-resonant energy gain predicted in the simulations. 
Jpn. J. Appl. Phys.

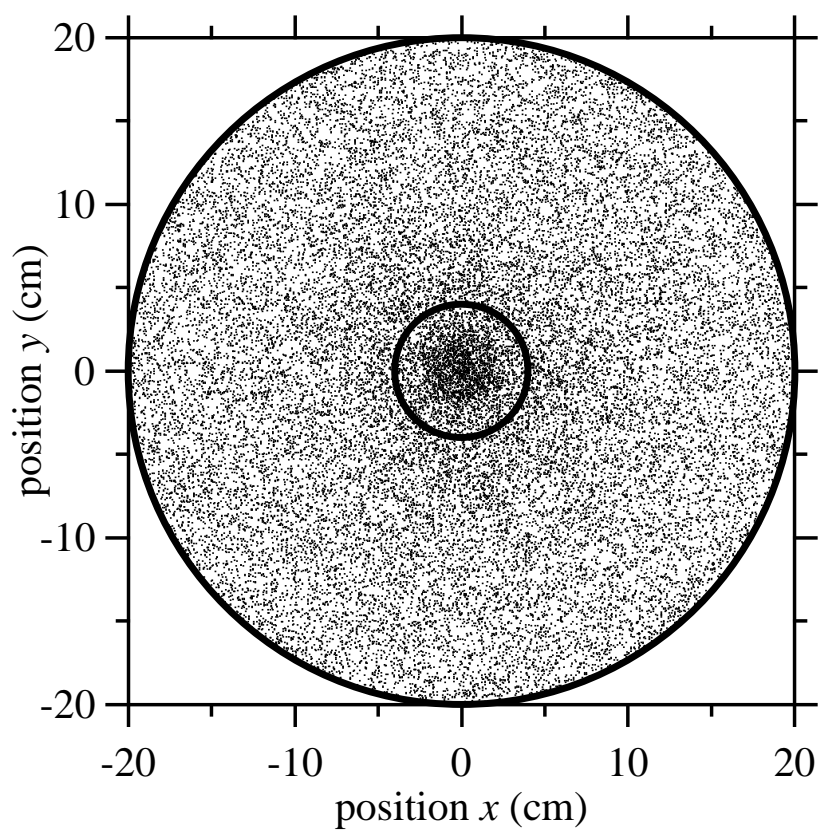

Fig. 7. Distribution of positions of electron passage across the separatrix during $t=0-100 T_{\mathrm{rf}}$. The initial electrons were released from the ceiling in the range of $r=0.0-5.0 \mathrm{~cm}$. Ar at $0.67 \mathrm{~Pa}$, $I_{\mathrm{dc}}=50.0 \mathrm{~A}$, and $I_{\mathrm{rf}}=10.0 \mathrm{~A}$. The inner circle indicates $r=r_{\mathrm{ECR}}$, where $|\boldsymbol{B}|=B_{\mathrm{ECR}}$.

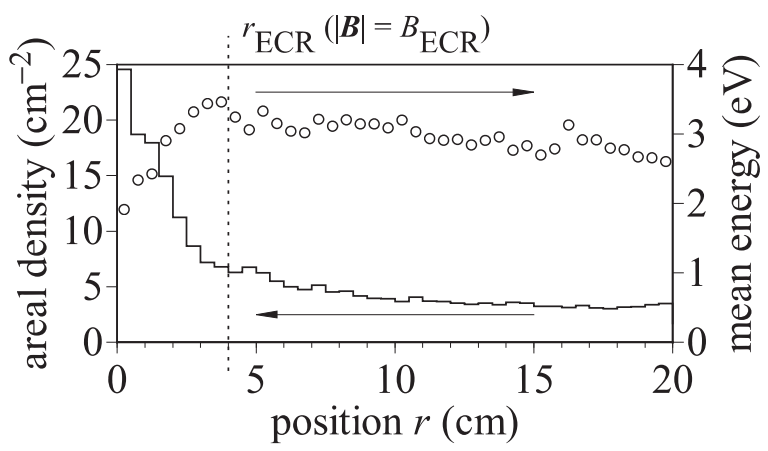

Fig. 8. Areal density of electron passages across the separatrix (histogram, normalized for 10000 samples) and mean energy at the passage (circles). Ar at $0.67 \mathrm{~Pa}, I_{\mathrm{dc}}=50.0 \mathrm{~A}$, and $I_{\mathrm{rf}}=10.0 \mathrm{~A}$.

\subsection{Electron passage across the separatrix}

Profiles of the electrons that passed across the separatrix were sampled under the following conditions. ${ }^{33}$ The gas was Ar at $0.67 \mathrm{~Pa}, I_{\mathrm{dc}}=50.0 \mathrm{~A}, I_{\mathrm{rf}}=10.0 \mathrm{~A}, r_{\mathrm{ref}}=1.00$, and $r_{\text {remain }}=$ 1.00. About 170000 electrons were released from the ceiling in the range of $r=0.0-5.0 \mathrm{~cm}$, and about 30000 of them passed across the separatrix by $t=100 T_{\mathrm{rf}}$. Figure 7 shows their first passage positions. Their areal density and the average energy at the passages as functions of $r$ are shown in Fig. 8. The areal density was normalized to values per 10000 samples.

Here, let us define 'the inner and outer separatrices' as two parts of the separatrix corre- 


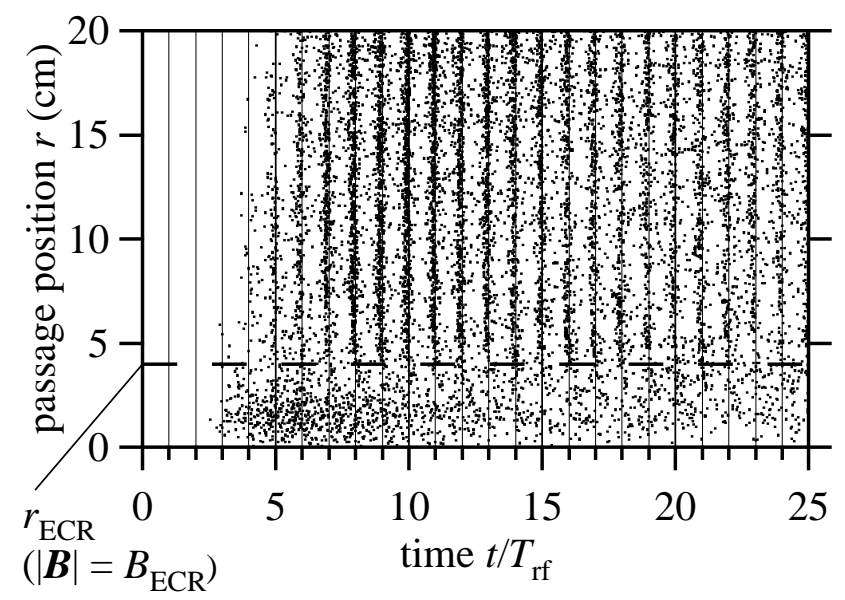

Fig. 9. Relationship between radial position $r$ and timing $t / T_{\text {rf }}$ of electron passage across the separatrix. Ar at $0.67 \mathrm{~Pa}, I_{\mathrm{dc}}=50.0 \mathrm{~A}$, and $I_{\mathrm{rf}}=10.0 \mathrm{~A}$.

sponding to the regions of $r<r_{\mathrm{ECR}}$ under the weak $\boldsymbol{B}$ field and $r>r_{\mathrm{ECR}}$ under the strong $\boldsymbol{B}$ field, respectively, for convenience in discussion. The electron passage positions in Fig. 7 concentrated around the X point. The inner separatrix seems to be a diffusion path to the lower region, as previously mentioned as the aperture of the separatrix. The mean energy of the electrons that passed this region was low. The weak $\boldsymbol{B}$ field allowed the passage of low-energy electrons. On the other hand, the mean energy at the outer separatrix was higher. It is considered that the electron passage across the outer separatrix is easier for electrons with larger gyroradii. A slight decay in the mean energy with $r$ indicates that passage across the separatrix by low-energy electrons becomes easier outward. In the outer region of large $r$, the magnetic flux lines approach the separatrix and even electrons with small gyroradii can pass the separatrix by the displacement of their gyrocenter due to scattering. This tendency was observed also in $\mathrm{H}_{2} \cdot{ }^{29}$ This position-dependent electron energy selectivity will be a key property of the magnetic filter.

Figure 9 shows the relationship between the position $r$ and the timing $t / T_{\mathrm{rf}}$ of the electron passages in early periods. The mode of electron passage was different between the inner and outer separatrices. The electron passage across the inner separatrix had no dependence on the phase of the rf $\boldsymbol{E}$ field. On the other hand, that across the outer separatrix concentrated around ends of rf periods. This indicates that the electron passage occurred also with the $\boldsymbol{E} \times \boldsymbol{B}$ drift. $^{33}$ The direction of the $\boldsymbol{E} \times \boldsymbol{B}$ drift at the separatrix is upward in the former half of the rf period and downward in the latter half in the present field configuration.

Primary factors of the electron transport across the separatrix of the CDMFs have been described. However, an argument is left: whether the judgement of the electron passage across the separatrix by the electron position is appropriate or not. Since gyrating electrons draw more or less circular trajectories, some of the electrons that passed the separatrix downward 
return to the upper region after their short stay in the lower region. The question is how we can distinguish this temporary stay from effective electron transfer to the lower region. A way to judge the substantial region to which an electron belongs would be judgement by the position of the gyrocenter instead, because the guiding magnetic flux line on which the gyrocenter locates determines the region of the electron motion. Nonetheless, even this judgement is still imperfect because the gyrocenter itself librates with the $\boldsymbol{E} \times \boldsymbol{B}$ drift. This issue is left for further investigation elsewhere.

\section{Conclusions}

The electron behavior under the CDMFs was simulated using a Monte Carlo method to investigate fundamental functions of the separatrix of the CDMFs as a magnetic shutter or filter.

The CDMFs guided electrons sideways, suppressing the electron diffusion across the separatrix. This function was demonstrated as a magnetic shutter. The electron confinement worked effectively under a strong $\boldsymbol{B}$ field. The electron confinement broke under a weak $\boldsymbol{B}$ field, but the separatrix showed a reverse-blocking effect resulting in one-way electron passage.

The electron passage across the separatrix showed two different modes. That across the outer separatrix was by scattering and the $\boldsymbol{E} \times \boldsymbol{B}$ drift, and that across the inner separatrix was by meandering.

The electron passage across the outer separatrix was pulselike because of the $\boldsymbol{E} \times \boldsymbol{B}$ drift alternating upward and downward synchronously to the $\mathrm{rf} \boldsymbol{E}$ field and was selective about electron energy. It was likely to occur for high-energy electrons with correspondingly large gyroradii. Low-energy electrons tended to be blocked by the outer separatrix. These effects would be used as a magnetic filter.

On the other hand, the inner separatrix was an aperture allowing electron passage. The aperture radius was defined by the position $r_{\mathrm{ECR}}$ of the rf-resonant magnetic field strength $B_{\mathrm{ECR}}$ and $r_{\mathrm{ECR}}$ was controllable via the coil currents. The electron passage through the aperture was continuous independently of the phase of the rf $\boldsymbol{E}$ field.

The position-dependent electron energy selectivity is a fundamental property of magnetic shutters and filters. The consideration of the arrangement of the separatrix and its aperture relative to a plasma enhancement source would be the next step for practical applications. For their reasonable design, we need to quantify some properties such as the selectivity and efficiency. In addition, an appropriate way to judge the electron transfer from one side of the separatrix to the other side is required. We expect that the fundamental properties and insights on the electron behavior under the CDMFs presented in this paper will become clues for resolving the remaining issues and lead to progress in the development of magnetic shutters and filters in future investigations. 
Jpn. J. Appl. Phys.

Regular Paper

\section{Acknowledgment}

This work was supported by a Grant-in-Aid No. 25400528 from the Japan Society for the Promotion of Science. 


\section{References}

1) M. M. M. Bilek, J. Appl. Phys. 85, 6385 (1999).

2) M. M. M. Bilek and I. G. Brown, IEEE Trans. Plasma Sci. 27, 193 (1999).

3) B. K. Tay, G. F. You, S. P. Lau, and X. Shi, Surf. Coatings Technol. 133-134, 593 (2000).

4) A. Anders, IEEE Trans. Plasma Sci. 30, 108 (2002).

5) E. Byon, J.-K. Kim, S.-C. Kwon, and A. Anders, IEEE Trans. Plasma Sci. 32, 433 (2004).

6) O. Fukumasa, Y. Tauchi, and S. Sakiyama, Jpn. J. Appl. Phys. 36, 4593 (1997).

7) N. Hayashi, T. Nakashima, and H. Fujita, Jpn. J. Appl. Phys. 38, 4301 (1999).

8) T. A. Santhosh Kumar, S. K. Mattoo, and R. Jha, Phys. Plasmas 11, 1735 (2004).

9) St. Kolev, St. Lishev, A. Shivarova, Kh. Tarnev, and R. Wilhelm, Plasma Phys. Control. Fusion 49, 1349 (2007).

10) M. Draghici and E. Stamate, J. Phys. D 43, 155205 (2010).

11) A. Aanesland, J. Bredin, P. Chabert, and V. Godyak, Appl. Phys. Lett. 100, 044102 (2012).

12) T. Tsankov and U. Czarnetzki, AIP Conf. Proc. 1390, 140 (2011).

13) Y. Celik, T. Tsankov, and U. Czarnetzki, IEEE Trans. Plasma Sci. 39, 2466 (2011).

14) T. Tsankov and U. Czarnetzki, IEEE Trans. Plasma Sci. 39, 2538 (2011).

15) T. V. Tsankov, K. Toko, and U. Czarnetzki, Phys. Plasmas 19, 123503 (2012).

16) A. Loarte, Plasma Phys. Control. Fusion 43, R183 (2001).

17) W. Chen, Y. Morikawa, M. Ito, T. Hayashi, K. Sugita, H. Shindo, and T. Uchida, J. Vac. Sci. Technol. A 17, 2546 (1999).

18) W. Chen, T. Hayashi, M. Ito, Y. Morikawa, K. Sugita, and T. Uchida, Vacuum 53, 29 (1999).

19) Y. Sakurai, T. Osaga, and H. Sugawara, IEEE Trans. Plasma Sci. 39, 2550 (2011).

20) T. Yoshida, Y. Sakurai, H. Sugawara, and A. Murayama, Denki Gakkai Ronbunshi A 132, 278 (2012) [in Japanese].

21) T. Yoshida, Y. Sakurai, H. Sugawara, and A. Murayama, Electr. Eng. Jpn. 185, 9 (2013).

22) Y. Asami and H. Sugawara, IEEE Trans. Plasma Sci. 42, 2540 (2014).

23) Y. Minami, Y. Asami, and H. Sugawara, IEEE Trans. Plasma Sci. 42, 2550 (2014).

24) H. Sugawara, N. Mori, Y. Sakai, and Y. Suda, J. Comput. Phys. 223, 298 (2007).

25) Y. Nakamura and M. Kurachi, J. Phys. D 21, 718 (1988).

26) P. T. Lan and B.-H. Jeon, J. Phys. Soc. Jpn. 81, 104501 (2012). 
27) H. Sugawara, J. Phys. Soc. Jpn. 82, 064501 (2013).

28) Z. Yoshida, H. Asakura, H. Kakuno, J. Morikawa, K. Takemura, S. Takizawa, and T. Uchida, Phys. Rev. Lett. 81, 2458 (1998).

29) H. Sugawara, Proc. 9th Int. Conf. Reactive Plasmas/ 68th Gaseous Electronics Conf./ 33rd Symp. Plasma Processing, 2015, KW2.00001.

30) H. Sugawara, Bull. Am. Phys. Soc. 60, 73 (2015).

31) T. Uchida, J. Vac. Sci. Technol. A 16, 1529 (1998).

32) T. Uchida and S. Hamaguchi, J. Phys. D 41, 083001 (2008).

33) H. Sugawara, Y. Minami, and S. Ogino, Proc. 22nd Europhysics Conf. Atomic and Molecular Physics of Ionized Gases, 2014, P1-02-04. 